\title{
On some relations between ideals of nowhere dense sets in topologies on positive integers
}

\author{
Andrzej Nowik ${ }^{1}$ Paulina Szyszkowska ${ }^{2}$
}

Accepted: 14 March 2021 / Published online: 30 August 2021

(c) The Author(s) 2021

\begin{abstract}
We examine the ideals of nowhere dense sets in three topologies on the set of positive integers, namely Furstenberg's, Rizza's and the common division topology. We mainly concentrate on inclusions between these ideals, we present a diagram showing these and we explore all possible inclusions between them. We present a formula for the closure of a set in the common division topology. We answer a question posed by Kwela and Nowik (Topol Appl. 248:149-163, 2018) by constructing a set in $\mathcal{I}_{G} \backslash\left(\mathcal{I}_{K} \cup \mathcal{I}_{F}\right)$. Therefore, the main diagram of comparison between the ideals of nowhere dense sets in various topologies from the article by M. Kwela and A. Nowik is completed.
\end{abstract}

Keywords Furstenberg's topology · Golomb's topology · Kirch's topology · Rizza's topology · Common division topology · Arithmetic progressions · Nowhere dense sets · Ideals

Mathematics Subject Classification 03E05 - 54D05 - 54H05 - 54A05 - 11A41 - 11B05 . 11B25

\section{Preliminaries}

The symbol $\mathbb{N}$ and Primes denote the set of positive integers and the set of primes, respectively. Let $[\mathbb{N}]^{\mathbb{N}}$ denote the collection of all infinite subsets of the set of natural numbers. For a subset $A$ of a topological space, we use the symbols $\operatorname{cl}(A)$ and $\operatorname{int}(A)$ for the closure and the interior of $A$, respectively. The symbols $\Theta(n)$ and $D(n)$ denote the set of all prime factors of $n$, (so $\Theta(n)=\{p \in$ Primes: $p \mid n\}$ ) and the set of all divisors of $n$, (so $D(n)=\{k \in \mathbb{N}: k \mid n\}$ ), respectively. Let $\mathbb{S F}$ denote the set of square-free numbers (i.e., numbers not divisible by any

$\varangle \quad$ Andrzej Nowik

andrzej@mat.ug.edu.pl

Paulina Szyszkowska

paulinaszczuka@wp.pl

1 Institute of Mathematics, University of Gdańsk, Wita Stwosza 57, 80-952 Gdańsk, Poland

2 Institute of Mathematics, Kazimierz Wielki University, Powstańców Wlkp. 2, 85-090 Bydgoszcz, Poland 
square greater than 1). Following [10], for all $a, b \in \mathbb{N}$, the symbol $\{a n+b\}$ stands for the infinite arithmetic progressions: $\{a n+b\}=\{a \cdot n+b: n \in \mathbb{N} \cup\{0\}\}$. Moreover, define an abbreviation: $\{a n\}=\{a n+a\}$. Let us say that a family $\mathcal{F} \subseteq[\mathbb{N}]^{\mathbb{N}}$ has the splitting property if for any $F \in \mathcal{F}$ one can find $F_{1}, F_{2} \in \mathcal{F}$ such that $F_{1} \cup F_{2} \subseteq F$ and $F_{1} \cap F_{2}=\emptyset$.

Let us define various topologies on $\mathbb{N}$ :

- Golomb's topology $(\mathbb{N}, \mathcal{D})$

with the base $\mathcal{B}_{G}=\{\{a n+b\}:(a, b)=1, b<a\}$,

- Kirch's topology $\left(\mathbb{N}, \mathcal{D}^{\prime}\right)$

with the base $\mathcal{B}_{K}=\{\{a n+b\}:(a, b)=1, b<a, a \in \mathbb{S F}\}$,

- Furstenberg's topology $\left(\mathbb{N}, \mathcal{T}_{F}\right)$

with the base $\mathcal{B}_{F}=\{\{a n+b\}: b \leq a\}$,

- the common division topology $(\mathbb{N}, \mathcal{T})$

with the base $\mathcal{B}_{\mathcal{T}}=\{\{a n+b\}: \Theta(a) \subseteq \Theta(b)\}$,

- the division topology (Rizza's topology) $\left(\mathbb{N}, \mathcal{T}^{\prime}\right)$

with the base $\mathcal{B}_{\mathcal{T}^{\prime}}=\{\{a n\}\}$.

Furstenberg's topology was first defined in [1] to present a topological proof of the existence of infinitely many prime numbers. This topology is metrizable, zero-dimensional and totally disconnected. Furstenberg's topology was originally defined on the set of integers but in this paper, in order to make our presentation more unified, we trim this topology to $\mathbb{N}$. Notice that the trimmed Furstenberg's topology is also metrizable, zero-dimensional and totally disconnected. Golomb's topology was defined in [2] to present a similar proof. Notice that Golomb's topology is Hausdorff but not regular. Kirch's topology was defined in [3] and this topology is again Hausdorff but not regular. Kirch's topology is weaker than Golomb's topology and it is locally connected, as opposed to Golomb's topology. Moreover, Rizza in [6] introduced the division topology. In [9] the author defined the common division topology $\mathcal{T}$ on $\mathbb{N}$, stronger than the division topology $\mathcal{T}^{\prime}$. Both topologies $\mathcal{T}$ and $\mathcal{T}^{\prime}$ are $T_{0}$, they are not $T_{1}$, and they are connected-however, the common division topology $\mathcal{T}$ is not locally connected, as opposed to the division topology $\mathcal{T}^{\prime}$. Let us notice that all these topologies have recently been studied by P. Szyszkowska née Szczuka, e.g., in [7,8,10].

An ideal on $\mathbb{N}$ is a family of subsets of $\mathbb{N}$ closed under taking finite unions and subsets of its elements. We assume that an ideal is proper and contains all finite sets. Obviously, in any $T_{1}$ topology without isolated points, the nowhere dense sets form an ideal. For each topology defined above, consider the ideal of nowhere dense sets: $\mathcal{I}_{G}, \mathcal{I}_{K}, \mathcal{I}_{F}, \mathcal{I}_{S}, \mathcal{I}_{R}$ in Golomb's, Kirch's, Furstenberg's, the common division, and Rizza's topology, respectively.

\section{Results}

In [5] the authors examined properties of the ideals $\mathcal{I}_{G}, \mathcal{I}_{K}$, and $\mathcal{I}_{F}$ and they asked if it is true that $\mathcal{I}_{G} \backslash\left(\mathcal{I}_{K} \cup \mathcal{I}_{F}\right) \neq \varnothing([5$, Problem 2.10]). It turns out that it is true. In the proof of the proposition below we present a simple solution of this problem.

Proposition $2.1 \mathcal{I}_{G} \backslash\left(\mathcal{I}_{K} \cup \mathcal{I}_{F}\right) \neq \emptyset$.

Proof We construct the set Ex as in the proof of [5, Theorem 2.9]. Namely, define

$$
C=\left\{\{a n+b\} \in \mathcal{B}_{K}:\{a n+b\} \subset\{2 n+1\}\right\} .
$$

Let $\left\{C_{k}: k \in \mathbb{N}\right\}$ be an enumeration of $C$. The set $E x=\left\{x_{k}: k \in \mathbb{N}\right\}$ is constructed as follows: for every $k \in \mathbb{N}$ we pick $x_{k}$ such that $x_{k} \in C_{k}$ and $x_{k} \in\left\{2^{k} n+1\right\}$. In the proof of 
[5, Theorem 2.9] it was shown that $E x \in\left(\mathcal{I}_{G} \cap \mathcal{I}_{F}\right) \backslash \mathcal{I}_{K}$. Now let

$$
X=\{2 n\} \cup E x .
$$

By [5, Example 2.6], $\{2 n\} \in \mathcal{I}_{G} \backslash \mathcal{I}_{F}$. So, $X \in \mathcal{I}_{G}$ as the sum of two nowhere dense sets in Golomb's topology, but $X \notin \mathcal{I}_{K}$ since $E x \notin \mathcal{I}_{K}$ and $X \notin \mathcal{I}_{F}$ since $\{2 n\} \notin \mathcal{I}_{F}$.

The diagram below is described in [5]. Our recent example (Proposition 2.1) can be seen in this diagram describing the relations between the ideals $\mathcal{I}_{K}, \mathcal{I}_{G}$ and $\mathcal{I}_{F}$. Hence, we finally completed the diagram.

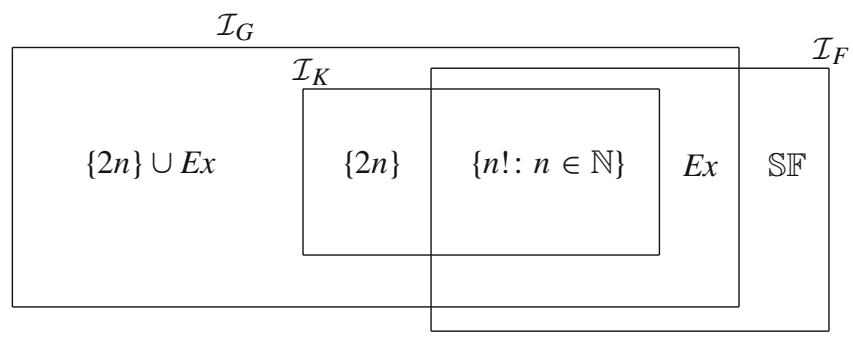

In this diagram, Ex is the set constructed in [5, Theorem 2.9].

In the next part of this paper we will mainly focus on the relationships between the ideals $\mathcal{I}_{R}, \mathcal{I}_{S}$ and $\mathcal{I}_{F}$. However, some relations between all five ideals will also be examined. At first, we present a proposition showing that Furstenberg's, Rizza's and the common division ideal are not disjoint.

Proposition 2.2 There is an infinite set in $\mathcal{I}_{R} \cap \mathcal{I}_{S} \cap \mathcal{I}_{F}$.

Proof Clearly

- Primes $\in \mathcal{I}_{S}([13$, p. 97]),

- Primes $\in \mathcal{I}_{R}$ since $\operatorname{int}_{\mathcal{T}^{\prime}}\left(\operatorname{cl}_{\mathcal{T}^{\prime}}(\right.$ Primes $\left.)\right)=\operatorname{int}_{\mathcal{T}^{\prime}}($ Primes $\cup\{1\})=\emptyset$,

- Primes $\in \mathcal{I}_{F}$ ([5, Example 2.2]).

Moreover,

$$
\left(\mathcal{I}_{F} \cap \mathcal{I}_{S} \cap \mathcal{I}_{R}\right) \backslash\left(\mathcal{I}_{G} \cup \mathcal{I}_{K}\right) \neq \emptyset
$$

since Primes $\notin \mathcal{I}_{G} \cup \mathcal{I}_{K}$ ([5, Example 2.2]).

The next propositions will show that the ideals $\mathcal{I}_{R}, \mathcal{I}_{S}$ and $\mathcal{I}_{F}$ differ significantly.

Proposition $2.3\left(\mathcal{I}_{R} \cap \mathcal{I}_{S}\right) \backslash \mathcal{I}_{F} \neq \varnothing$.

Proof We will show $\{2 n+1\} \in\left(\mathcal{I}_{R} \cap \mathcal{I}_{S}\right) \backslash \mathcal{I}_{F}$. First observe that $\{2 n+1\} \notin \mathcal{I}_{F}$. Moreover,

- $\operatorname{int}_{\mathcal{T}^{\prime}}\left(\operatorname{cl}_{\mathcal{T}^{\prime}}(\{2 n+1\})\right)=\operatorname{int}_{\mathcal{T}^{\prime}}(\{2 n+1\})=\emptyset$ (we clearly have $\forall_{\substack{U \in \mathcal{T}^{\prime} \\ U \neq \emptyset}} U \cap\{2 n\} \neq \emptyset$, which implies $\left.\forall_{\substack{U \in \mathcal{T}^{\prime} \\ U \neq \emptyset}} U \backslash\{2 n+1\} \neq \emptyset\right)$.

- $\operatorname{int}_{\mathcal{T}}\left(\operatorname{cl}_{\mathcal{T}}(\{2 n+1\})\right)=\operatorname{int}_{\mathcal{T}}(\{2 n+1\})=\emptyset$ since

Claim 2.4 $\forall_{\substack{U \in \mathcal{T} \\ U \neq \emptyset}} U \cap\{2 n\} \neq \emptyset$. 
Indeed, if $U \in \mathcal{T} \backslash\{\emptyset\}$, then we can find $\{a n+b\} \subseteq U$ such that $\Theta(a) \subseteq \Theta(b)$. Let us consider the following cases:

$a, b$ even: then $\{a n+b\} \cap\{2 n\} \neq \emptyset$,

$a, b$ odd: then $2 \mid(a+b)$ so again $\{a n+b\} \cap\{2 n\} \neq \emptyset$,

$a$ even; $b$ odd: impossible, since $\Theta(a) \subseteq \Theta(b)$,

$a$ odd; $b$ even: then $2 \mid(2 a+b)$, hence $\{a n+b\} \cap\{2 n\} \neq \emptyset$.

So, $\underset{\substack{U \in \mathcal{T} \\ U \neq \emptyset}}{\forall} U \backslash\{2 n+1\} \neq \emptyset$. Consequently, $\{2 n+1\} \in \mathcal{I}_{R} \cap \mathcal{I}_{S}$.

Moreover,

$$
\left(\mathcal{I}_{R} \cap \mathcal{I}_{S}\right) \backslash\left(\mathcal{I}_{F} \cup \mathcal{I}_{G} \cup \mathcal{I}_{K}\right) \neq \varnothing .
$$

This follows from $\{2 n+1\} \in \mathcal{D}$ and $\{2 n+1\} \in \mathcal{D}^{\prime}$. Therefore $\{2 n+1\} \notin \mathcal{I}_{G}$ and $\{2 n+1\} \notin \mathcal{I}_{K}$.

Remark 2.5 If $A \notin \mathcal{I}_{R}$, then $A$ is dense in $\left(\mathbb{N}, \mathcal{T}^{\prime}\right)$.

Proof If $A \notin \mathcal{I}_{R}$, then $\operatorname{int}_{\mathcal{T}^{\prime}}\left(\operatorname{cl}_{\mathcal{T}^{\prime}}(A)\right) \neq \emptyset$. So $\{a n\} \subseteq \operatorname{cl}_{\mathcal{T}^{\prime}}(A)$. By [12, Corollary 6.5], $\mathbb{N}=\operatorname{cl}_{\mathcal{T}^{\prime}}(\{a n\}) \subseteq \operatorname{cl}_{\mathcal{T}^{\prime}}(A)$.

Corollary 2.6 (Independently proved in [4]) In the division (Rizza's) topology all sets are either dense or nowhere dense.

Proposition $2.7 \mathcal{I}_{R} \backslash\left(\mathcal{I}_{S} \cup \mathcal{I}_{F}\right) \neq \emptyset$.

Proof We will show $\{4 n+2\} \in \mathcal{I}_{R} \backslash\left(\mathcal{I}_{S} \cup \mathcal{I}_{F}\right)$. Indeed, since $\{4 n+2\}$ is open in $\mathcal{T}$ and in $\mathcal{T}_{F},\{4 n+2\} \notin \mathcal{I}_{S} \cup \mathcal{I}_{F}$. By Remark 2.5, either $\{4 n+2\} \in \mathcal{I}_{R}$ or $\{4 n+2\}$ is dense in $\left(\mathbb{N}, \mathcal{T}^{\prime}\right)$ but it cannot be dense as $\{4 n+2\} \cap\{4 n\}=\emptyset$ and $\{4 n\} \in \mathcal{T}^{\prime}$.

Moreover,

$$
\left(\mathcal{I}_{R} \cap \mathcal{I}_{G} \cap \mathcal{I}_{K}\right) \backslash\left(\mathcal{I}_{S} \cup \mathcal{I}_{F}\right) \neq \varnothing .
$$

Since $\mathcal{I}_{K} \subseteq \mathcal{I}_{G}$ ([5, Theorem 2.5]), it is sufficient to show $\{4 n+2\} \in \mathcal{I}_{K}$. By $\left[11\right.$, Theorem 4.3], $\operatorname{cl}_{\mathcal{D}^{\prime}}(\{4 n+2\})=\{2 n\}$, hence

$$
\operatorname{int}_{\mathcal{D}^{\prime}}\left(\operatorname{cl}_{\mathcal{D}^{\prime}}(\{4 n+2\})\right)=\operatorname{int}_{\mathcal{D}^{\prime}}(\{2 n\})=\emptyset .
$$

Indeed, if $\operatorname{int}_{\mathcal{D}^{\prime}}(\{2 n\}) \neq \emptyset$, then there would exist $\{a n+b\} \in \mathcal{B}_{K}$ such that $\{a n+b\} \subseteq\{2 n\}$. Consider the following cases:

$a$ even: then $\{a n+b\} \subseteq\{2 n+1\}$ (notice that $(a, b)=1)$, a contradiction,

$a$ even; $b$ odd: then $a+b \notin\{2 n\}$, impossible,

$a, b$ even: then $2 a+b \notin\{2 n\}$, impossible.

To prove the next proposition we will need the characterization of closed sets in the common division topology.

Remark 2.8 There is a characterization of the closure of a set in the division topology, namely: $\operatorname{cl}_{\mathcal{T}^{\prime}}(A)=\bigcup_{a \in A} D(a)($ see $[6])$.

Let us formulate an analogous characterization for the common division topology:

Theorem $2.9 \operatorname{cl}_{\mathcal{T}}(A)=\left\{x \in \mathbb{N}: \forall_{k \in \mathbb{N}} \exists_{a_{k} \in A} a_{k} \equiv x\left(\bmod x^{k}\right)\right\}$. 
Proof Write $B=\left\{x \in \mathbb{N}: \forall_{k \in \mathbb{N}} \exists_{a_{k} \in A} a_{k} \equiv x\left(\bmod x^{k}\right)\right\}$. We have to prove $B=\operatorname{cl}_{\mathcal{T}}(A)$. Observe that $x \in B$ is equivalent to $\forall_{k \in \mathbb{N}} \exists_{a_{k} \in A} a_{k} \in\left\{x^{k} n+x\right\}$ which in turn is equivalent to $\forall_{k \in \mathbb{N}} A \cap\left\{x^{k} n+x\right\} \neq \emptyset$.

If $x \notin B$, then $\exists_{k \in \mathbb{N}} A \cap\left\{x^{k} n+x\right\}=\emptyset$. Since $x \in\left\{x^{k} n+x\right\}$ and $\left\{x^{k} n+x\right\} \in \mathcal{T}$, we have $x \notin \operatorname{cl}_{\mathcal{T}}(A)$.

Now suppose $x \in B$. If $x=1$, then by [9, Proposition 3.1] $x \in \operatorname{cl}_{\mathcal{T}}(A)$. So, we can suppose $x \neq 1$. Let us choose $U \in \mathcal{T}$ such that $x \in U$. By [10, Lemma 3.1], there exists $\{c n+x\} \in \mathcal{B}_{\mathcal{T}}$ such that $\{c n+x\} \subseteq U$. Since $\{c n+x\} \in \mathcal{B}_{\mathcal{T}}$, we have $\Theta(c) \subseteq \Theta(x)$. Let $x=p_{1}^{\alpha_{1}} \cdots p_{m}^{\alpha_{m}}$ be a prime factor decomposition of $x$. Since $\Theta(c) \subseteq \Theta(x)$, without loss of generality we may assume that $c=p_{1}^{\beta_{1}} \cdots p_{i}^{\beta_{i}}$ is a prime factor decomposition of $c$, where $1 \leq i \leq m$. Let $k=\Pi_{j=1}^{i} \beta_{j}$. Then $c \mid x^{k}$, hence $\left\{x^{k} n+x\right\} \subseteq\{c n+x\}$. But since $\left\{x^{k} n+x\right\} \cap A \neq \emptyset$, we have $U \cap A \supseteq\{c n+x\} \cap A \supseteq\left\{x^{k} n+x\right\} \cap A \neq \emptyset$. Thus, $x \in \operatorname{cl}_{\mathcal{T}}(A)$.

Corollary $2.10 x \in \operatorname{cl}_{\mathcal{T}}(A)$ if and only if $\forall_{k \in \mathbb{N}} A \cap\left\{x^{k} n+x\right\} \neq \emptyset$.

Corollary 2.11 If $A \subseteq \mathbb{N}$ is a finite set, then $\operatorname{cl}_{\mathcal{T}}(A)=A \cup\{1\}$.

Proof Let $x \notin A \cup\{1\}$. Since $A$ is a finite set, $\exists_{k \in \mathbb{N}} \forall_{a \in A} x^{k}>a$. Then obviously $\left\{x^{k} n+\right.$ $x\} \cap A=\emptyset$. By Corollary $2.10, x \notin \mathrm{cl}_{\mathcal{T}}(A)$.

On the other hand, $A \subseteq \operatorname{cl}_{\mathcal{T}}(A)$ and since $\forall_{k \in \mathbb{N}} A \cap\left\{1^{k} n+1\right\}=A \cap \mathbb{N}=A \neq \emptyset$, again by Corollary $2.10,1 \in \operatorname{cl}_{\mathcal{T}}(A)$.

Proposition $2.12\left(\mathcal{I}_{S} \cap \mathcal{I}_{F}\right) \backslash \mathcal{I}_{R} \neq \emptyset$.

Proof Let $A=\{n !: n \in \mathbb{N}\}$. By [5, Example 2.1], $A \in \mathcal{I}_{F}$. Now we will show $A \notin \mathcal{I}_{R}$. Observe that $\operatorname{cl}_{\mathcal{T}^{\prime}}(A)=\mathbb{N}$. Indeed, fix $x \in \mathbb{N}$ and define $a=x$ !. Then, by Remark 2.8, $x \in D(a) \subset \bigcup_{a^{\prime} \in A} D\left(a^{\prime}\right)=\operatorname{cl}_{\mathcal{T}^{\prime}}(A)$. Therefore $A \notin \mathcal{I}_{R}$.

Finally, we have to show $A \in \mathcal{I}_{S}$. At first we show that $A$ is a closed set in $(\mathbb{N}, \mathcal{T})$. Suppose $x \in \operatorname{cl}_{\mathcal{T}}(A) \backslash A$. By Corollary 2.10, $\forall_{k \in \mathbb{N}} A \cap\left\{x^{k} n+x\right\} \neq \emptyset$. With $k=2$ we obtain $A \cap\left\{x^{2} n+x\right\} \neq \emptyset$. Define $A_{1}=\left\{n !: n=1, \ldots, x^{2}-1\right\}$ and $A_{2}=A \backslash A_{1}$. Since $\operatorname{cl}_{\mathcal{T}}(A)=\operatorname{cl}_{\mathcal{T}}\left(A_{1}\right) \cup \operatorname{cl}_{\mathcal{T}}\left(A_{2}\right)$ and $x \notin A_{1}=A_{1} \cup\{1\}=\operatorname{cl}_{\mathcal{T}}\left(A_{1}\right)$ (by virtue of Corollary 2.11), so $x \in \operatorname{cl}_{\mathcal{T}}\left(A_{2}\right)$. Observe that $A_{2} \subseteq\left\{x^{2} n\right\}$, and since $x \in \operatorname{cl}_{\mathcal{T}}\left(A_{2}\right)$, by Corollary 2.10, we deduce $A_{2} \cap\left\{x^{2} n+x\right\} \neq \emptyset$, which is impossible, since $\left\{x^{2} n\right\} \cap\left\{x^{2} n+x\right\}=\emptyset$.

Next, $\operatorname{int}_{\mathcal{T}}\left(\operatorname{cl}_{\mathcal{T}}(A)\right)=\operatorname{int}_{\mathcal{T}}(A)=\emptyset$, which yields $A \in \mathcal{I}_{S}$.

Moreover, we obtain

$$
\left(\mathcal{I}_{S} \cap \mathcal{I}_{F} \cap \mathcal{I}_{G} \cap \mathcal{I}_{K}\right) \backslash \mathcal{I}_{R} \neq \emptyset .
$$

This follows from [5, Example 2.1], where it was shown that $A=\{n !: n \in \mathbb{N}\} \in \mathcal{I}_{G} \cap \mathcal{I}_{K}$.

Proposition $2.13 \mathcal{I}_{S} \backslash\left(\mathcal{I}_{R} \cup \mathcal{I}_{F}\right) \neq \emptyset$.

Proof Let $A=\{n !: n \in \mathbb{N}\}$ and $X=A \cup\{2 n+1\}$. Then $X \in \mathcal{I}_{S}$, since $A \in \mathcal{I}_{S}$ and $\{2 n+1\} \in \mathcal{I}_{S}$. Observe that $X \notin \mathcal{I}_{R}$ since $A \notin \mathcal{I}_{R}$ and $X \notin \mathcal{I}_{F}$ since $\{2 n+1\} \notin \mathcal{I}_{F}$ (cf. the proofs of Proposition 2.12 and Proposition 2.3).

Moreover,

$$
\mathcal{I}_{S} \backslash\left(\mathcal{I}_{F} \cap \mathcal{I}_{G} \cap \mathcal{I}_{K} \cap \mathcal{I}_{R}\right) \neq \emptyset .
$$

This follows from $\{2 n+1\} \notin \mathcal{I}_{K} \cup \mathcal{I}_{G}$.

All relations among the ideals $\mathcal{I}_{S}, \mathcal{I}_{R}$ and $\mathcal{I}_{F}$ proven in this article can be seen in the following diagram: 


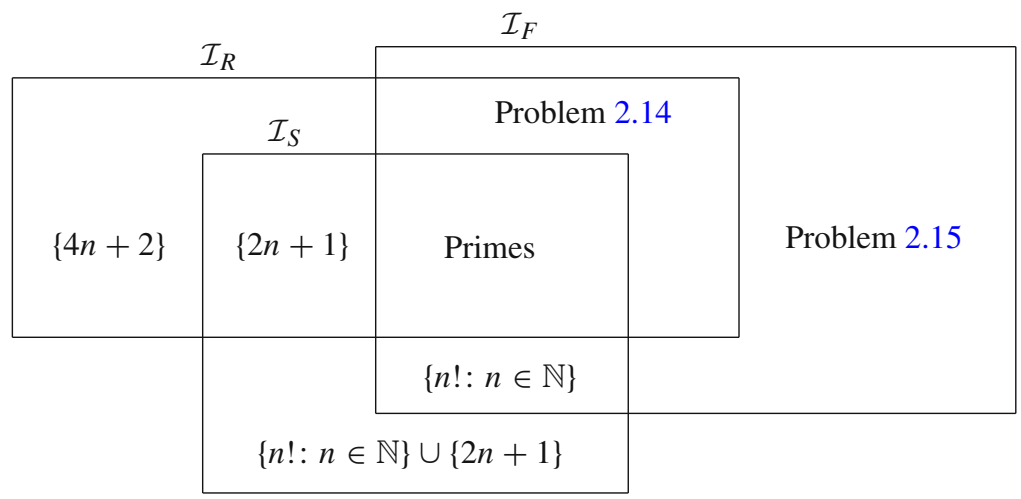

Problem 2.14 Is it true that $\left(\mathcal{I}_{R} \cap \mathcal{I}_{F}\right) \backslash \mathcal{I}_{S} \neq \varnothing$ ?

Problem 2.15 Is it true that $\mathcal{I}_{F} \backslash\left(\mathcal{I}_{R} \cup \mathcal{I}_{S}\right) \neq \emptyset$ ?

Finally, note that if the answer to Problem 2.14 is positive, then the answer to Problem 2.15 will also be positive.

Let us end the article with a result about the splitting property of the common division topology.

Namely, by Proposition 1.1 from [5] we know that any base for any Hausdorff topology without isolated points has the splitting property. However, the division and the common division topology is not Hausdorff, therefore it is natural to ask whether these two topologies have the splitting property. We obtain

Proposition 2.16 The family $\mathcal{B}_{\mathcal{T}}$ has the splitting property.

Proof Suppose $\{a n+b\} \in \mathcal{B}_{\mathcal{T}}$. Then $\Theta(a) \subseteq \Theta(b)$. Consider two cases:

$a>1$ : Define $F_{1}=\left\{a^{2} n+b\right\}$ and $F_{2}=\left\{a^{2} n+(a+b)\right\}$.

$a=1$ : Define $F_{1}=\left\{(b+1)^{2} n\right\}$ and $F_{2}=\left\{(b+1)^{2} n+(b+1)\right\}$.

Then in both cases $F_{1}, F_{2} \in \mathcal{B}_{\mathcal{T}}, F_{1} \cap F_{2}=\emptyset$ and $F_{1} \cup F_{2} \subseteq\{a n+b\}$.

On the other hand, the family $\mathcal{B}_{\mathcal{T}}$, does not have the splitting property, since $a b \in\{a n\} \cap\{b n\}$.

Open Access This article is licensed under a Creative Commons Attribution 4.0 International License, which permits use, sharing, adaptation, distribution and reproduction in any medium or format, as long as you give appropriate credit to the original author(s) and the source, provide a link to the Creative Commons licence, and indicate if changes were made. The images or other third party material in this article are included in the article's Creative Commons licence, unless indicated otherwise in a credit line to the material. If material is not included in the article's Creative Commons licence and your intended use is not permitted by statutory regulation or exceeds the permitted use, you will need to obtain permission directly from the copyright holder. To view a copy of this licence, visit http://creativecommons.org/licenses/by/4.0/.

\section{References}

1. H. Furstenberg, On the infinitude of primes. Am. Math. Mon. 62(5), 353 (1955) 
2. S.W. Golomb, A connected topology for the integers. Am. Math. Mon. 66(8), 663-665 (1959)

3. A.M. Kirch, A countable, connected, locally connected Hausdorff space. Am. Math. Mon. 76(2), 169-171 (1969)

4. M. Kwela, Rizza's ideal and comparison of some known set-theoretic ideals from number theory and combinatorics. Preprint

5. M. Kwela, A. Nowik, Ideals of nowhere dense sets in some topologies on positive integers. Topol. Appl. 248, 149-163 (2018)

6. G.B. Rizza, A topology for the set of non-negative integers. Riv. Mat. Univ. Parma 5(2), 179-185 (1993)

7. P. Szczuka, The connectedness of arithmetic progressions in Furstenberg's, Golomb's and Kirch's topologies. Demonstr. Math. 43(4), 899-909 (2010)

8. P. Szczuka, The Darboux property for polynomials in Golomb's and Kirch's topologies. Demonstr. Math. 46(2), 429-435 (2013)

9. P. Szczuka, Connections between connected topological spaces on the set of positive integers. Cent. Eur. J. Math. 11(5), 876-881 (2013)

10. P. Szczuka, The closures of arithmetic progressions in the common division topology on the set of positive integers. Cent. Eur. J. Math. 12(7), 1008-1014 (2014)

11. P. Szczuka, The closures of arithmetic progressions in Kirch's topology on the set of positive integers. Int. J. Number Theory 11(3), 673-682 (2015)

12. P. Szczuka, Properties of the division topology on the set of positive integers. Int. J. Number Theory 12(3), 775-786 (2016)

13. P. Szyszkowska, M. Szyszkowski, Properties of the common division topology on the set of positive integers. J. Ramanujan Math. Soc. 33(1), 91-98 (2018)

Publisher's Note Springer Nature remains neutral with regard to jurisdictional claims in published maps and institutional affiliations. 